\title{
OPTIMAL LINEAR FILTERING WITH STATE AND OBSERVATION DELAYS
}

\author{
Michael Basin * Aracelia Alcorta-Garcia* \\ Jesus Rodriguez-Gonzalez*
}

\author{
* Department of Physical and Mathematical Sciences \\ Autonomous University of Nuevo Leon, Mexico
}

\begin{abstract}
In this paper, the optimal filtering problem for linear systems with state and observation delays is treated proceeding from the general expression for the stochastic Ito differential of the optimal estimate, error variance, and various error covariances. As a result, the optimal estimate equation similar to the traditional Kalman-Bucy one is derived; however, the resulting system of equations for determining the filter gain matrix consists, in the general case, of an infinite set of equations. It is then demonstrated that a finite set of the filtering equations, whose number is specified by the ratio between the current filtering horizon and the delay values, can be obtained in the particular case of equal or commensurable delays in the observation and state equations. In the example, performance of the designed optimal filter for linear systems with state and observation delays is verified against the best KalmanBucy filter available for linear systems without delays. Copyright (C)2005 IFAC.
\end{abstract}

Keywords: Filtering, stochastic system, linear time-delay system

\section{INTRODUCTION}

The optimal filtering problem for linear system states and observations without delays was solved in 1960s (Kalman and Bucy, 1961), and this closed form solution is known as the Kalman-Bucy filter. However, the related optimal filtering problem for linear states with delay has not been solved in a closed form, regarding as a closed form solution a closed system of a finite number of ordinary differential equations for any finite filtering horizon. The optimal filtering problem for time delay systems itself did not receive so much attention as its control counterpart, and most of the research was concentrated on the filtering problems with observation delays (the papers (Alexander, 1991; Hsiao and Pan, 1996; Larsen et al., 1998) could be mentioned to make a reference). A particular case, the optimal filtering problem for linear systems with multiple observation delays, has recently been solved in (Basin and Martinez-Zuniga, 2004).

\footnotetext{
1 This work was supported by the Mexican National Science and Technology Council (CONACyT) under Grant 39388-A.
}

A review of the bibliography on dual optimal control problems, as well as robust filtering and control problems, for time delay systems can be found in (Basin and Martinez-Zuniga, 2004; Basin et al., 2003; Basin et al., 2004a). Comprehensive reviews of general theory and algorithms for time delay systems are given in (Kolmanovskii and Shaikhet, 1996; Kolmanovskii and Myshkis, 1999; Malek-Zavarei and Jashmidi, 1987; Mahmoud, 2000; Boukas and Liu, 2002).

In this paper, the optimal filtering problem for linear systems with state and observation delays is treated proceeding from the general expression for the stochastic Ito differential of the optimal estimate, error variance, and various error covariances (Pugachev and Sinitsyn, 2001). As a result, the optimal estimate equation similar to the traditional Kalman-Bucy one is derived. However, it is impossible to obtain a system of the filtering equations, that is closed with respect to the only two variables, the optimal estimate and the error variance, as in the Kalman-Bucy filter. Thus, the resulting system of equations for determining the filter gain matrix consists, in the general case, of an infinite 
set of equations. It is however demonstrated that a finite set of the filtering equations can be obtained in the particular case of equal or commensurable $(\tau=q h)$ delays in the observation and state equations, where $\tau$ is the observation delay, $h$ is the state one, and $q$ is a natural number. This finite number of the filtering equations whose number is specified by the ratio between the current filtering horizon and the delay values and increases as the filtering horizon tends to infinity.

The paper is organized as follows. Section 2 and 3 present the filtering problem statement for a linear system with state and observation delays and its solution, respectively. In Section 4, performance of the obtained optimal filter for linear systems with state and observation delays is verified in the illustrative example against the best filter available for linear systems without delays. The simulation results show asymptotic convergence of the estimate given by the obtained optimal filter for linear systems with state and observation delays to the real system state as time tends to infinity, whereas the conventional KalmanBucy estimates calculated without delay adjustment do not converge.

\section{FILTERING PROBLEM FOR LINEAR SYSTEMS WITH STATE AND OBSERVATION DELAYS}

Let $(\Omega, F, P)$ be a complete probability space with an increasing right-continuous family of $\sigma$-algebras $F_{t}, t \geq 0$, and let $\left(W_{1}(t), F_{t}, t \geq 0\right)$ and $\left(W_{2}(t), F_{t}, t \geq\right.$ $0)$ be independent Wiener processes. The partially observed $F_{t}$-measurable random process $(x(t), y(t))$ is described by a delay differential equation for the system state

$$
d x(t)=\left(a_{0}(t)+a(t) x(t-h)\right) d t+b(t) d W_{1}(t),
$$

with the initial condition $x(s)=\phi(s), s \in\left[t_{0}-h, t_{0}\right]$, and a delay differential equation for the observation process

$$
d y(t)=\left(A_{0}(t)+A(t) x(t-\tau)\right) d t+B(t) d W_{2}(t),
$$

where $x(t) \in R^{n}$ is the state vector, $y(t) \in R^{m}$ is the observation process, $\phi(s)$ is a mean square piecewisecontinuous Gaussian stochastic process (see (Pugachev and Sinitsyn, 2001) for definition) given in the interval $\left[t_{0}-h, t_{0}\right]$ such that $\phi(s), W_{1}(t)$, and $W_{2}(t)$ are independent. The system state $x(t)$ dynamics depends on a delayed state $x(t-h)$ and the observations $y(t)$ are collected depending on another delayed state $x(t-\tau)$, which actually make the system state space infinitedimensional (see, for example, (Malek-Zavarei and Jashmidi, 1987)). The vector-valued function $a_{0}(t)$ describes the effect of system inputs (controls and disturbances). It is assumed that $A(t)$ is a nonzero matrix and $B(t) B^{T}(t)$ is a positive definite matrix. All coefficients in (1)-(2) are deterministic functions of appropriate dimensions.
The estimation problem is to find the best estimate of the system state $x(t)$ based on the observation process $Y(t)=\{y(s), 0 \leq s \leq t\}$, which minimizes the Euclidean 2-norm

$$
J=E\left[(x(t)-\hat{x}(t))^{T}(x(t)-\hat{x}(t)) \mid F_{t}^{Y}\right]
$$

at every time moment $t$. Here, $E\left[z(t) \mid F_{t}^{Y}\right]$ means the conditional expectation of a stochastic process $z(t)=(x(t)-\hat{x}(t))^{T}(x(t)-\hat{x}(t))$ with respect to the $\sigma$ - algebra $F_{t}^{Y}$ generated by the observation process $Y(t)$ in the interval $\left[t_{0}, t\right]$. As known (Pugachev and Sinitsyn, 2001), this optimal estimate is given by the conditional expectation

$$
\hat{x}(t)=m(t)=E\left(x(t) \mid F_{t}^{Y}\right)
$$

of the system state $x(t)$ with respect to the observation process $Y(t)$ in the interval $\left[t_{0}, t\right]$.

The matrix functions $P(t)=$

$$
=E\left[(x(t)-m(t))(x(t)-m(t))^{T} \mid F_{t}^{Y}\right],
$$

that is the estimation error variance, and $P\left(t, t-t_{1}\right)=$

$$
=E\left[(x(t)-m(t))\left(x\left(t-t_{1}\right)-m\left(t-t_{1}\right)\right)^{T} \mid F_{t}^{Y}\right],
$$

that is the covariance between the estimation error values at different time moments, $P(t, t)=P(t)$, are used to obtain a system of filtering equations.

The proposed solution to this optimal filtering problem is based on the formulas for the Ito differentials of the conditional expectation $m(t)=E\left(x(t) \mid F_{t}^{Y}\right)$, the error variance $P(t)$, and other bilinear functions of $x(t)-m(t)$ (see (Pugachev and Sinitsyn, 2001)) and given in the following section.

\section{OPTIMAL FILTER FOR LINEAR SYSTEMS WITH STATE AND OBSERVATION DELAYS}

The optimal filtering equations can be obtained using the formula for the Ito differential of the conditional expectation $m(t)=E\left(x(t) \mid F_{t}^{Y}\right)(($ Pugachev and Sinitsyn, 2001))

$$
\begin{gathered}
d m(t)=E\left(\varphi(x) \mid F_{t}^{Y}\right) d t+ \\
E\left(x\left[\varphi_{1}(x)-E\left(\varphi_{1}(x) \mid F_{t}^{Y}\right)\right]^{T} \mid F_{t}^{Y}\right) \times \\
\left(B(t) B^{T}(t)\right)^{-1}\left(d y(t)-E\left(\varphi_{1}(x) \mid F_{t}^{Y}\right) d t\right),
\end{gathered}
$$

where $\varphi(x)$ is the drift term in the state equation equal to $\varphi(x)=a_{0}(t)+a(t) x(t-h)$ and $\varphi_{1}(x)$ is the drift term in the observation equation equal to $\varphi_{1}(x)=$ $A_{0}(t)+A(t) x(t-\tau)$. Note that the conditional expectation equality $E\left(x(t-h) \mid F_{t}^{Y}\right)=E\left(x(t-h) \mid F_{t-h}^{Y}\right)=$ $m(t-h)$ is valid for any $h>0$, since, in view of a positive delay shift $h>0$, the treated problem (1),(2) is a filtering problem, not a smoothing one, and, therefore, the formula (3) yields the optimal estimate $m(s)$ for any time $s, t_{0}<s \leq t$, if the observations (2) are obtained until the current moment $t$ (see (Pugachev and Sinitsyn, 2001; Basin and Martinez-Zuniga, 2004)). Upon performing substitution of the expressions for $\varphi$ 
and $\varphi_{1}$ into (3) and taking into account the conditional expectation equality, the estimate equation takes the form

$$
\begin{gathered}
d m(t)=\left(a_{0}(t)+a(t) m(t-h)\right) d t+ \\
E\left(x(t)[A(t)(x(t-\tau)-m(t-\tau))]^{T} \mid F_{t}^{Y}\right) \times \\
\left(B(t) B^{T}(t)\right)^{-1}\left(d y(t)-\left(A_{0}(t)+A(t) m(t-\tau) d t\right)=\right. \\
=\left(a_{0}(t)+a(t) m(t-h)\right) d t+ \\
E\left([x(t)-m(t)][x(t-\tau)-m(t-\tau)]^{T} \mid F_{t}^{Y}\right) A^{T}(t) \times \\
\left(B(t) B^{T}(t)\right)^{-1}\left(d y(t)-\left(A_{0}(t)+A(t) m(t-\tau) d t\right)=\right. \\
\left(a_{0}(t)+a(t) m(t-h)\right) d t+P(t, t-\tau) A^{T}(t) \times \\
\left(B(t) B^{T}(t)\right)^{-1}\left(d y(t)-\left(A_{0}(t)+A(t) m(t-\tau) d t\right)\right.
\end{gathered}
$$

To compose a system of the filtering equations, the equation for the conditional expectation $E([x(t)-$ $\left.m(t)][(x(t-\tau)-m(t-\tau))]^{T} \mid F_{t}^{Y}\right)$ should be obtained. This can be done using the equation (1) for the state $x(t)$, the equation (4) for the estimate $m(t)$, and the formula for the Ito differential of a product of two processes satisfying Ito differential equations ((Pugachev and Sinitsyn, 2001)):

$$
\begin{gathered}
d\left(z_{1} z_{2}^{T}\right)=z_{1} d z_{2}^{T}+\left(z_{2} d z_{1}^{T}\right)^{T}+ \\
(1 / 2)\left[y_{1} v y_{2}^{T}+y_{2} v y_{1}^{T}\right] d t .
\end{gathered}
$$

Here, the stochastic process $z_{1}$ satisfies the equation $d z_{1}=x_{1} d t+y_{1} d w_{1}$, the stochastic process $z_{2}$ satisfies the equation $d z_{2}=x_{2} d t+y_{2} d w_{2}$, and $v$ is the covariance intensity matrix of the Wiener vector $\left[w_{1} w_{2}\right]^{T}$.

Let us obtain the formula for the Ito differential of the general expression $P\left(t, t-t_{1}\right)=E([x(t)-m(t)][x(t-$ $\left.\left.\left.t_{1}\right)-m\left(t-t_{1}\right)\right]^{T} \mid F_{t}^{Y}\right)$, where $t_{1}>0$ is an arbitrary delay, not necessarily equal to $\tau$. Upon representing $P\left(t, t-t_{1}\right)$ as $P\left(t, t-t_{1}\right)=E\left(\left[x(t)\left(x\left(t-t_{1}\right)\right)^{T}\right] \mid F_{t}^{Y}\right)-$ $m(t) m\left(t-t_{1}\right)$, using first $x(t)$ as $z_{1}$ and $x\left(t-t_{1}\right)$ as $z_{2}$ and then $m(t)$ as $z_{1}$ and $m\left(t-t_{1}\right)$ as $z_{2}$ in the formula (5), taking into account independence of the Wiener processes $W_{1}$ and $W_{2}$ in the equations (1) and (2), and finally subtracting the second derived equation from the first one, the following formula is obtained

$$
\begin{gathered}
d P\left(t, t-t_{1}\right) / d t=a(t) P\left(t-h, t-t_{1}\right) \\
+P\left(t, t-t_{1}-h\right) a^{T}\left(t-t_{1}\right) \\
+(1 / 2)\left[b(t) b^{T}\left(t-t_{1}\right)+b\left(t-t_{1}\right) b^{T}(t)\right. \\
-P(t, t-\tau) A^{T}(t)\left(B(t) B^{T}(t)\right)^{-1} B(t) B^{T}\left(t-t_{1}\right) \\
\times\left(B\left(t-t_{1}\right) B^{T}\left(t-t_{1}\right)\right)^{-1} A\left(t-t_{1}\right) P^{T}\left(t-t_{1}, t-t_{1}-\tau\right) \\
-P\left(t-t_{1}, t-t_{1}-\tau\right) A^{T}\left(t-t_{1}\right)\left(B\left(t-t_{1}\right) B^{T}\left(t-t_{1}\right)\right)^{-1} \\
\left.\times B\left(t-t_{1}\right) B^{T}(t)\left(B(t) B^{T}(t)\right)^{-1} A(t) P^{T}(t, t-\tau)\right] .
\end{gathered}
$$

Analysis of the formula (6) in the case $t_{1}=\tau$ implies that the equation for $P(t, t-\tau)$ includes variables $P(t, t-\tau-h), P(t-h, t-\tau)$ and the same $P(t, t-$ $\tau)$ in its right-hand side. Taking into account that $P(t-h, t-\tau)$ is represented as $P(t, t-\tau+h)$ with the arguments delayed by $h$, the new variables involved in the equations for $P(t, t-\tau)$ are $P(t, t-\tau-h)$ and $P(t, t-\tau+h)$. This structure is repeated in the equations for $P(t, t-\tau-h), P(t, t-\tau+h)$, etc.
Hence, the system of the optimal filtering equations for the state (1), whose proper dynamics is delayed by $h$, over the delayed by $\tau$ observations (2) is the infinite-dimensional system composed by the equation (4) for the optimal estimate and the equations (6) for the covariances $P(t, t-\tau+k h)$, where $k=$ $\ldots,-2,-1,0,1,2, \ldots$ is an arbitrary integer number.

Using the notation $P_{k}(t)=P(t, t-\tau-k h)$, the equation (4) can be rewritten as

$$
\begin{gathered}
d m(t)=\left(a_{0}(t)+a(t) m(t-h)\right) d t+P_{0}(t) A^{T}(t) \times( \\
\left(B(t) B^{T}(t)\right)^{-1}\left(d y(t)-\left(A_{0}(t)+A(t) m(t-\tau) d t\right),\right.
\end{gathered}
$$

and the system (6) can be represented in the following form

$$
\begin{gathered}
d P_{k}(t) / d t=a(t) P_{k-1}(t-h)+P_{k+1}(t) a^{T}(t-\tau-k h) \\
+(1 / 2)\left[b(t) b^{T}(t-\tau-k h)+b(t-\tau-k h) b^{T}(t)\right. \\
-P_{0}(t) A^{T}(t)\left(B(t) B^{T}(t)\right)^{-1} B(t) B^{T}(t-\tau-k h) \\
\times\left(B(t-\tau-k h) B^{T}(t-\tau-k h)\right)^{-1} A(t-\tau-k h) \\
\times P_{0}^{T}(t-\tau-k h)-P_{0}(t-\tau-k h) A^{T}(t-\tau-k h) \\
\quad \times\left(B(t-\tau-k h) B^{T}(t-\tau-k h)\right)^{-1} \\
\left.\quad \times B(t-\tau-k h) B^{T}(t)\left(B(t) B^{T}(t)\right)^{-1} A(t) P_{0}^{T}(t)\right] .
\end{gathered}
$$

Thus, the preceding conclusion can be formulated in the final form: the system of the optimal filtering equations for the state (1), whose proper dynamics is delayed by $h$, over the delayed by $\tau$ observations (2) is the infinite system composed by the equation (7) for the optimal estimate and the equations (8) for the covariances $P_{k}(t)=P(t, t-\tau-k h)$, where $k=$ $\ldots,-2,-1,0,1,2, \ldots$ is an arbitrary integer number.

The last step is to establish the initial conditions for the system of equations (7),(8). The initial conditions for (7) are stated as

$$
\begin{gathered}
m(s)=E(\phi(s)), s \in\left[t_{0}-h, t_{0}\right) \text { and } \\
m\left(t_{0}\right)=E\left(\phi\left(t_{0}\right) \mid F_{t_{0}}^{Y}\right), s=t_{0},
\end{gathered}
$$

The initial conditions for matrices $P_{k}(t)=E((x(t)-$ $\left.m(t))(x(t-\tau-k h))^{T} \mid F_{t}^{Y}\right)$ should be stated as functions in the intervals $\left[\max \left\{t_{0}-h, t_{0}+\tau+(k-1) h\right\}\right.$, $\left.\max \left\{t_{0}+\tau+k h, t_{0}\right\}\right]$, since the equations (8) corresponding to non-negative $k$ depend on coefficients with arguments delayed by $\tau+k h$, which are not defined for $t<t_{0}$. Thus, the initial conditions for the matrices $P_{k}(t)$ are stated as

$$
\begin{gathered}
P_{k}(s)=E((x(s)-m(s))(x(s-\tau-k h)-\quad(10) \\
\left.m(s-\tau-k h))^{T} \mid F_{s}^{Y}\right), \quad s \in \\
{\left[\max \left\{t_{0}-h, t_{0}+\tau+(k-1) h\right\}, \max \left\{t_{0}+\tau+k h, t_{0}\right\}\right] .}
\end{gathered}
$$

Unfortunately, the system (7),(8) cannot be reduced to a finite system for any fixed filtering horizon $t$, as it can be done in the case of only state delay in the equations (1),(2) (see (Basin et al., 2004b)), since the infinite number of the equations (8) for $P_{k}(t)$ with negative $k$ are always needed to compose a closed system for any 
time $t$. However, this reduction is possible for some particular cases, for example, in the case of equal, $\tau=h$, (or commensurable, $\tau=q h, q$ is natural) delays in the equations (1),(2), which is considered in details in the next subsection.

\subsection{Optimal Filter for Linear Systems with Commensurable State and Observation Delays}

An important and frequently encountered in practical applications particular case of commensurable delays in state and observation equations is recovered assuming $\tau=q h, q=1,2, \ldots$ is a natural number. In doing so, the state and observation equations (1),(2) take the form

$$
d x(t)=\left(a_{0}(t)+a(t) x(t-h)\right) d t+b(t) d W_{1}(t),
$$

with the initial condition $x(s)=\phi(s), s \in\left[t_{0}-h, t_{0}\right]$,

$$
d y(t)=\left(A_{0}(t)+A(t) x(t-q h)\right) d t+B(t) d W_{2}(t) .
$$

Accordingly, the optimal filtering equation (7) for the optimal estimate $m(t)$ turns to

$$
\begin{gathered}
d m(t)=\left(a_{0}(t)+a(t) m(t-h)\right) d t+P_{0}(t) A^{T}(t) \times \\
\left(B(t) B^{T}(t)\right)^{-1}\left(d y(t)-\left(A_{0}(t)+A(t) m(t-q h) d t\right),\right.
\end{gathered}
$$

and the system (8) is given by

$$
\begin{gathered}
d P_{k}(t) / d t=a(t) P_{k-1}(t-h)+P_{k+1}(t) a^{T}(t-(q+k) h) \\
+(1 / 2)\left[b(t) b^{T}(t-(q+k) h)+b(t-(q+k) h) b^{T}(t)\right. \\
-P_{0}(t) A^{T}(t)\left(B(t) B^{T}(t)\right)^{-1} B(t) B^{T}(t-(q+k) h) \\
\times\left(B(t-(q+k) h) B^{T}(t-(q+k) h)\right)^{-1} \\
\times A(t-(q+k) h) P_{0}^{T}(t-(q+k) h) \\
-P_{0}(t-(q+k) h) A^{T}(t-(q+k) h) \\
\times\left(B(t-(q+k) h) B^{T}(t-(q+k) h)\right)^{-1} \\
\left.\times B(t-(q+k) h) B^{T}(t)\left(B(t) B^{T}(t)\right)^{-1} A(t) P_{0}^{T}(t)\right] .
\end{gathered}
$$

Using the equality

$$
\begin{gathered}
P_{-q-1}(t-h)=P(t-h, t-(-q-1) h-q h-h)= \\
P(t-h, t)=P^{T}(t, t-h)=P_{-q+1}^{T}(t),
\end{gathered}
$$

the equation for $P_{-q}$ in (14) can be rewritten as

$$
\begin{gathered}
d P_{-q}(t) / d t=a(t) P_{-q+1}^{T}(t)+P_{-q+1}(t) a^{T}(t)+ \\
b(t) b^{T}(t)-P_{0}(t) A^{T}(t)\left(B(t) B^{T}(t)^{-1} A(t) P_{0}^{T}(t) .\right.
\end{gathered}
$$

Note that $P_{-q}(t)=E\left((x(t)-m(t))(x(t))^{T} \mid F_{t}^{Y}\right)$ is the estimation error variance.

If $q=1,2, \ldots$, the right-hand side of (15) does not include variables $P_{k}$ corresponding to negative $k<-q$. Hence, a closed system of the filtering equations is formed by the equations (13),(15) and the equations (14) with $k \geq-q$ only. This enables one to obtain a finite system of the filtering equations for any fixed filtering horizon $t$, as follows.
Namely, for every fixed $t$, the number of equations corresponding to $k \geq-q$ in (14), that should be taken into account to obtain a closed system of the filtering equations, is not equal to infinity, since the matrices $a(t), b(t), A(t)$, and $B(t)$ are not defined for $t<t_{0}$. Therefore, if the current time moment $t$ belongs to the semi-open interval $\left(t_{0}+(k+q) h, t_{0}+(k+q+1) h\right]$, where $h$ is the delay value in the equations (1),(2), the number of equations in (14) is equal to $k+q$.

The last step is to establish the initial conditions for the system of equations (13),(15),(14). The initial conditions for (13) and (15) are stated as

$$
\begin{gathered}
m(s)=E(\phi(s)), s \in\left[t_{0}-\tau, t_{0}\right) \text { and } \\
m\left(t_{0}\right)=E\left(\phi\left(t_{0}\right) \mid F_{t_{0}}^{Y}\right), s=t_{0},
\end{gathered}
$$

and

$$
P\left(t_{0}\right)=E\left[\left(x\left(t_{0}\right)-m\left(t_{0}\right)\left(x\left(t_{0}\right)-m\left(t_{0}\right)\right)^{T} \mid F_{t_{0}}^{Y}\right] .\right.
$$

The initial conditions for matrices $P_{k}(t)=E((x(t)-$ $\left.m(t))(x(t-(q+k) h))^{T} \mid F_{t}^{Y}\right)$ should be stated as functions in the intervals $\left[t_{0}+(k+q-1) h, t_{0}+(k+q) h\right]$, since the $k$ th of the equations (14) depends on functions with the arguments delayed by $(k+q) h$ and the definition of $P_{k}(t)$ itself assumes dependence on $x(t-$ $(k+q) h)$. Thus, the initial conditions for the matrices $P_{k}(t)$ in (14) are stated as

$$
\begin{gathered}
P_{k}(s)=E((x(s)-m(s))(x(s-(q+k) h)- \\
\left.m(s-(q+k) h))^{T} \mid F_{s}^{Y}\right), \\
s \in\left[t_{0}+(q+k-1) h, t_{0}+(q+k) h\right] .
\end{gathered}
$$

The obtained system of the filtering equations (13), (15), (14) with the initial conditions (16)-(18) presents the optimal solution to the filtering problem for the linear state with delay (11) over the linear observations (12). A considerable advantage of the designed filter is a finite number of the filtering equations for any fixed filtering horizon, although the state space of the delayed system (11) is infinite-dimensional.

Remark. The convergence properties of the obtained optimal estimate (7) are given by the standard convergence theorem (see, for example, (Jazwinski, 1970)): if in the system (1),(2) the pair $(a(t) \Psi(t-h, t), b(t))$ is uniformly completely controllable and the pair $(a(t) \Psi(t-h, t), A(t) \Psi(t-\tau, t)$ is uniformly completely observable, where $\Psi(t, \tau)$ is the state transition matrix for the equation (1) (see (Malek-Zavarei and Jashmidi, 1987) for definition of matrix $\Psi$ ), then the error of the obtained optimal filter (7),(8) is uniformly asymptotically stable. As usual, the uniform complete controllability condition is required for assuring nonnegativeness of the error variance matrix $P_{-q}(t)$ and may be omitted, if the matrix $P_{-q}(t)$ is non-negative in view of its intrinsic properties. The uniform complete controllability and observability conditions for a linear system with delay (1) and observations (2) can be found in (Malek-Zavarei and Jashmidi, 1987). 


\section{EXAMPLE}

This section presents an example of designing the optimal filter for linear systems with state and observation delays and comparing it to the best filter available for linear systems without delay, that is the KalmanBucy filter (Kalman and Bucy, 1961).

Let the unobserved state $x(t)$ with delay be given by

$$
\dot{x}(t)=x(t-5), \quad x(s)=\phi(s), s \in[-5,0],
$$

where $\phi(s)=N(0,1)$ for $s \leq 0$, and $N(0,1)$ is a Gaussian random variable with zero mean and unit variance. The observation process is given by

$$
y(t)=x(t-5)+\psi(t),
$$

where $\psi(t)$ is a white Gaussian noise, which is the weak mean square derivative of a standard Wiener process (see (Pugachev and Sinitsyn, 2001)). The equations (12) and (13) present the conventional form for the equations (1) and (2), which is actually used in practice (Åström, 1970). Since the observation delay is equal to the state one, the system (19),(20) satisfies the conditions of Subsection 3.1 with $q=1$.

The filtering problem is to find the optimal estimate for the linear state with delay (19), using the linear observations with delay (20) confused with independent and identically distributed disturbances modeled as white Gaussian noises. Let us set the filtering horizon time to $T=80$. Since $80 \in(15 \times 5,16 \times 5]$, where 5 is the delay value in the equations (19),(20), the first 15 of the equations (14), along with the equations (13) and (15), should be employed.

The filtering equations (13),(15), and the first 15 of the equations (14) take the following particular form for the system (19),(20)

$$
\dot{m}(t)=m(t-5)+P_{0}(t)[y(t)-m(t-5)],
$$

with the initial condition $m(s)=E(\phi(s))=0, s \in$ $[-5,0)$ and $m(0)=E(\phi(0) \mid y(0))=m_{0}, s=0$;

$$
\dot{P}_{i}(t)=P_{i-1}(t-5)+P_{i+1}(t)-P_{0}(t) P_{0}(t-5(i+1)) \text {, }
$$

with the initial condition $P_{i}(0)=E((x(s)-m(s))(x(s-$ $\left.5(i+1))-m(s-5(i+1))) \mid F_{s}^{Y}\right), s \in[5 i, 5(i+1)]$, $i=0, \ldots, 14$; and

$$
\dot{P}_{-1}(t)=2 P_{0}(t)-P_{0}^{2}(t),
$$

with the initial condition $P_{-1}(0)=E\left((x(s)-m(s))^{2} \mid\right.$ $y(0))=R_{0}$; note that $P_{-1}(t)$ is the error variance. The particular forms of the equations (19) and (21) and the initial condition for $x(t)$ imply that $P_{i}(s)=R_{0}$, $i=0, \ldots, 15$, for $s \in[5 i, 5(i+1)]$.

The estimates obtained upon solving the equations (21)-(23) are compared to the conventional KalmanBucy estimates satisfying the following filtering equations for the linear state with delay (19) over linear observations with delay (20), where the variance equation is a Riccati one and the equation for matrix $P_{0}(t)$ is not employed:

$$
\dot{m}_{K}(t)=m_{K}(t-5)+P_{K}(t)\left[y(t)-m_{K}(t-5)\right],
$$

with the initial condition $m_{K}(s)=E(\phi(s))=0, s \in$ $[-5,0)$ and $m_{K}(0)=E(\phi(0) \mid y(0))=m_{0}, s=0$;

$$
\dot{P}_{K}(t)=2 P_{K}(t)-P_{K}^{2}(t),
$$

with the initial condition $P_{K}(0)=E\left((x(0)-m(0))^{2} \mid\right.$ $y(0))=R_{0}$.

Numerical simulation results are obtained solving the systems of filtering equations (21)-(23) and (24)-(25). The obtained values of the estimates $m(t)$ and $m_{K}(t)$ satisfying (21) and (24) respectively are compared to the real values of the state variable $x(t)$ in (19).

For each of the two filters (21)-(23) and (24)-(25) and the reference system (19) involved in simulation, the following initial values are assigned: $x_{0}=1, m_{0}=10$, $R_{0}=100$. Gaussian disturbance $\psi(t)$ in (20) is realized using the built-in MatLab white noise function.

The following graphs are obtained: graphs of the reference state variable $x(t)$ for the system (19); graphs of the Kalman-Bucy filter estimate $m_{K}(t)$ satisfying the equations (24)-(25); graphs of the optimal filter estimate for linear systems with state and observation delays $m(t)$ satisfying the equations (21)-(23). The graphs of all those variables are shown on the entire simulation interval from $T=0$ to $T=80$ (Fig. 1), and around the reference time points: $T=40$ (Fig. 2), $T=$ 60 (Fig. 3), and $T=80$ (Fig. 4). The following values of the reference state variable $x(t)$ and the estimates $m(t)$ and $m_{K}(t)$ are obtained at the reference time points: for $T=40, x(40)=12.55, m(40)=12.62$, $m_{K}(40)=12.75$; for $T=60, x(60)=51.56, m(60)=$ $51.50, m_{K}(60)=52.12$; for $T=80, x(80)=211.92$, $m(80)=211.96, m_{K}(80)=214.08$.

Thus, it can be concluded that the obtained optimal filter for a linear systems with state delay and over linear observations with delay (21)-(23) yield better estimates than the conventional Kalman-Bucy filter. Moreover, it can be seen that the estimate produced by the optimal filter for a linear state with delay over linear observations asymptotically converges to the real values of the reference variable as time tends to infinity, although the reference system (19) itself is unstable. On the contrary, the conventionally designed (non-optimal) Kalman-Bucy estimates do not converge to the real values. This significant improvement in the estimate behavior is obtained due to the more careful selection of the filter gain matrix using the multi-equational system (21)-(23), which compensates for unstable dynamics of the reference system, as it should be in the optimal filter.

\section{REFERENCES}

Alexander, H. L. (1991). State estimation for distributed systems with sensing delay. SPIE. Data Structures and Target Classification.

Åström, K. J. (1970). Introduction to Stochastic Control Theory. Vol. 70 of Mathematics in Science and Engineering. Academic Press. New York. 
Basin, M. V. and R. Martinez-Zuniga (2004). Optimal linear filtering over observations with multiple delays. International Journal of Robust and Nonlinear Control 14, 685-696.

Basin, M. V., J. G. Rodriguez-Gonzalez and R. Martinez-Zuniga (2004a). Optimal control for linear systems with time delay in control input. Journal of The Franklin Institute 341, 267-278.

Basin, M. V., J. G. Rodriguez-Gonzalez and R. Martinez-Zuniga (2004b). Optimal filtering for linear state delay systems, submitted to. IEEE Transactions on Automatic Control.

Basin, M. V., L. M. Fridman, P. Acosta and J. G. Rodriguez-Gonzalez (2003). Optimal and robust sliding mode control for linear systems with multiple time delays in control input. Asian Journal of Control 5, 557-567.

Boukas, E.-K. and Z.-K. Liu (2002). Deterministic and Stochastic Time-Delay Systems. Birkhauser.

Hsiao, F. H. and S. T. Pan (1996). Robust Kalman filter synthesis for uncertain multiple time-delay stochastic systems. ASME Transactions. J. of Dynamic Systems, Measurement, and Control 118, 803-807.

Jazwinski, A. H. (1970). Stochastic Processes and Filtering Theory. Academic Press. New York.

Kalman, R. E. and R. S. Bucy (1961). New results in linear filtering and prediction theory. ASME Journal of Basic Engineering, Ser. D 83, 95-108.

Kolmanovskii, V. B. and A. D. Myshkis (1999). Introduction to the Theory and Applications of Functional Differential Equations. Kluwer. New York.

Kolmanovskii, V. B. and L. E. Shaikhet (1996). Control of Systems with Aftereffect. American Mathematical Society. Providence.

Larsen, T. D., N. A. Andersen, O. Ravn and N. K. Poulsen (1998). Incorporation of the timedelayed measurements in a discrete-time Kalman filter. In: Proc. 37th Conf. on Decision and Control. pp. 3972-3977.

Mahmoud, M. S. (2000). Robust Control and Filtering for Time-Delay Systems. Marcel Dekker.

Malek-Zavarei, M. and M. Jashmidi (1987). TimeDelay Systems: Analysis, Optimization and Applications. North-Holland. Amsterdam.

Pugachev, V. S. and I. N. Sinitsyn (2001). Stochastic Systems: Theory and Applications. World Scientific. Singapore.
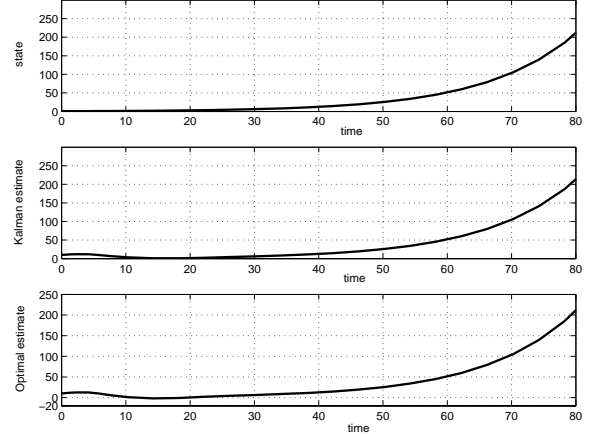

Figure 1. Graphs of the reference state variable $x(t)$ and the estimates $m_{K}(t)$ and $m(t)$ on the entire simulation interval $[0,80]$.
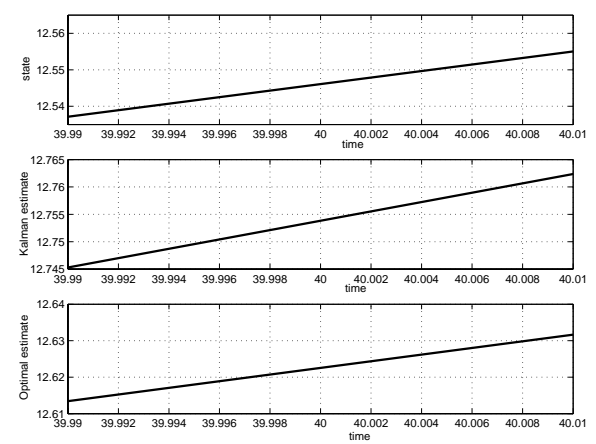

Figure 2. Graphs of the reference state variable $x(t)$ and the estimates $m_{K}(t)$ and $m(t)$ around the reference time point $T=40$.
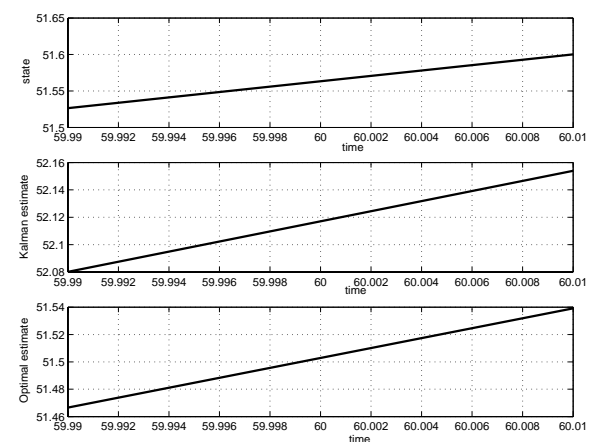

Figure 3. Graphs of the reference state variable $x(t)$ and the estimates $m_{K}(t)$ and $m(t)$ around the reference time point $T=60$.
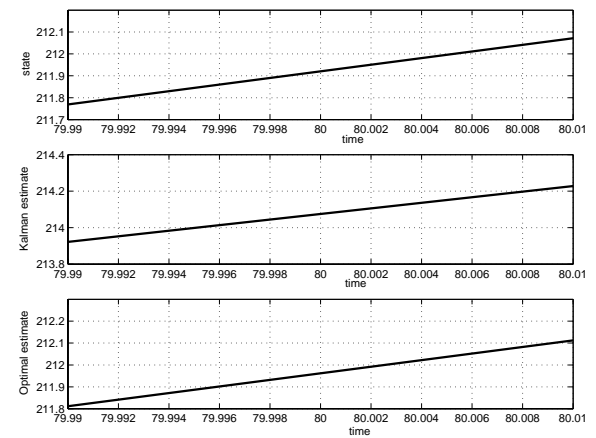

Figure 4. Graphs of the reference state variable $x(t)$ and the estimates $m_{K}(t)$ and $m(t)$ around the reference time point $T=80$. 